delayed neurodevelopment, whereas those who experienced CNS involvement without cardiopulmonary failure had good neurodevelopmental outcomes. Cognitive function was found to correlate with both the clinical severity of CNS infection and age at onset.

The effects of EV71 infection on neurological development and cognitive function seem to be strongly associated with the severity of CNS involvement and the patient's age at disease onset. Chang et al. conclude that these parameters should be used when assessing the long-term prognosis of viral CNS infection.

Original article Chang L-Y et al. (2007) Neurodevelopment and cognition in children after enterovirus 71 infection.

N Engl J Med 356: 1226-1234

\section{Inhibition of angiogenesis with celecoxib prevents germinal matrix hemorrhage}

Most cases of germinal matrix hemorrhage $(\mathrm{GMH})$ occur within the first $24 \mathrm{~h}$ following birth, regardless of gestational age. In a recently published study, Ballabh et al. assessed the role of vascular immaturity in $\mathrm{GMH}$, and tested in rabbits a potential new approach to preventing brain hemorrhage in preterm infants.

The investigators theorized that an increased rate of angiogenesis in the germinal matrix leads to instability of the developing vasculature and thereby increases the risk of hemorrhage occurring shortly after birth. Ballabh et al. confirmed that levels of vascular endothelial growth factor (VEGF) and angiopoietin-2 (ANGP2) were elevated in the germinal matrix compared with other areas of the developing brain, and also that the germinal matrix was a site of significantly increased endothelial proliferation. These patterns were seen in the brains of human fetuses, preterm infants and premature rabbit pups. To test the hypothesis that inhibition of angiogenesis would lower the incidence of $\mathrm{GMH}$, the authors administered celecoxib, an inhibitor of VEGF and ANGP2, to pregnant rabbits. Compared with controls, angiogenesis was suppressed in the preterm rabbit pups treated with celecoxib, and importantly the incidence and severity of GMH significantly decreased $(P<0.01$ for both) in the celecoxib-treated group.
The results of this study indicate that celecoxib administered to mothers in preterm labor might prevent $\mathrm{GMH}$; however, this application would of course need to be tested in clinical trials.

Original article Ballabh P et al. (2007) Angiogenic inhibition reduces germinal matrix hemorrhage. Nat Med 13: $477-485$

\section{Temozolomide is an active agent for the treatment of primary brain lymphomas}

Methotrexate-based chemotherapy is the firstline treatment for patients with primary brain lymphoma; however, in most cases this disease remains incurable. The treatment options for patients with recurrent primary brain lymphoma are limited, and so far there is little evidence to indicate that multi-agent chemotherapy is superior to methotrexate alone. Reni and coauthors have recently assessed the activity of the alkylating agent temozolomide in patients with relapsed or refractory primary brain lymphoma.

This phase II study included 36 patients with a median age of 60 years (range 34-81 years), who had been diagnosed with primary brain lymphoma and had previously failed to respond to high-dose methotrexate and/or radiotherapy treatment. The patients received $150 \mathrm{mg} / \mathrm{m}^{2}$ of temozolomide daily for 5 days every 4 weeks, for a maximum of six cycles. This salvage treatment yielded nine complete responses and two partial responses (response rate $31 \%$, $95 \% \mathrm{Cl} 16-46 \%)$. The median overall survival was 3.9 months (interquartile range 1.716 months), and the median progression-free survival was 2.8 months (interquartile range 1-8 months). The 1-year overall survival was $31 \%(95 \% \mathrm{Cl} 16-46 \%)$. The drug was generally well-tolerated, with two patients experiencing grade 4 neutropenia, and one patient experiencing grade 3 vomiting in a single cycle of treatment.

The authors conclude that temozolomide shows potential in the treatment of primary brain lymphomas, and suggest that further studies of this agent are warranted.

Original article Reni M et al. (2007) Temozolomide as salvage treatment in primary brain lymphomas. $\mathrm{Br}$ J Cancer 96: $864-867$ 